\title{
Linear analytical approach to dispersive, external dissipative, and intrinsic dissipative couplings in optomechanical systems
}

\author{
J. Baraillon $\odot$, B. Taurel, P. Labeye, and L. Duraffourg $\odot^{*}$ \\ Université Grenoble Alpes, Commissariat à l'Energie Atomique, LETI, F38054 Grenoble, France
}

(Received 7 April 2020; accepted 8 July 2020; published 11 September 2020)

\begin{abstract}
This paper presents a theoretical study of optomechanical systems in which the mechanical resonator modulates both the resonant frequency (dispersive coupling) and the decay rates (dissipative coupling) of the optical cavity. The generic dispersive framework is extended to a more general case in which the dissipative coupling is split between its external and intrinsic contribution. We report a complete analysis of the influence of both external and intrinsic optical losses on each of the three coupling mechanisms, highlighting the interest of each optical loss regime. A presentation of the basic model to experimentally identify the three couplings and their relative influence on the optical response is proposed. We also extend the basic tools by analyzing the mechanical dynamics and demonstrating the general expression of the optical spring effect and of optomechanical damping. A comparison between our theoretical model and experimental measurements in photonic crystal systems from the literature yields good agreement.
\end{abstract}

DOI: 10.1103/PhysRevA.102.033509

\section{INTRODUCTION}

Cavity optomechanics explores the mutual interaction of electromagnetic radiation and mechanical vibrations. In most optomechanical systems, the mechanical displacement modulates the resonant frequency of an optical cavity. This coupling of dispersive nature gives rise to several phenomena, such as the optical spring effect and the amplification or cooling of the mechanical motion. These have been studied theoretically [1-3], and have been achieved in various setups such as a membrane inside a Fabry-Pérot cavity (so-called membranein-the-middle) [4], suspended microdisks [5], and phoxonic crystal systems [6]. Additionally, dissipative coupling, where the photon decay rate is modulated by mechanical vibrations, can also arise in optomechanical systems. This scheme was first proposed theoretically in the context of optomechanical cooling as an alternative to dispersive coupling in the so-called unresolved sideband limit [7], and also for its squeezing ability [8-10]. This coupling mechanism was implemented experimentally in diverse configurations such as Michelson-Sagnac interferometers [11-14], whispering gallery mode resonators coupled to a nanomechanical beam waveguide [15-18], ring resonators coupled to a micromechanical resonator [19], and photonic crystal $(\mathrm{PhC})$ systems $[20,21]$.

More recently, it has been experimentally shown for two different PhC systems [20-22] that dissipative coupling must be split into its external $\left(\kappa_{\mathrm{om}}^{e}\right)$ and intrinsic $\left(\kappa_{\mathrm{om}}^{i}\right)$ contributions. $\mathrm{Wu}$ et al., in their PhC split-beam nanocavity [Fig. 1(a)], performed measures of dispersive coupling $\left(g_{\mathrm{om}}\right)$ and $\kappa_{\mathrm{om}}^{i}$ in the $\mathrm{GHz} / \mathrm{nm}$ range and $\kappa_{\mathrm{om}}^{e}$ in the $\mathrm{MHz} / \mathrm{nm}$ range [20].

\footnotetext{
*laurent.duraffourg@cea.fr

Published by the American Physical Society under the terms of the Creative Commons Attribution 4.0 International license. Further distribution of this work must maintain attribution to the author(s) and the published article's title, journal citation, and DOI.
}

While the external dissipative coupling is weaker in absolute terms, they observed that it has a larger relative impact in the undercoupled regime (low optical losses at the input drive port $\bar{\kappa}_{e}$ compared to the intracavity losses $\bar{\kappa}_{i}$ ), which means that the optical loss regime (defined by the ratio $\bar{\kappa}_{e} / \bar{\kappa}_{i}$ ) is likely to have a strong influence on the optomechanical response. In addition to this experimental study, Tsvirkun et al., in their PhC slab suspended over an input waveguide [Fig. 1(b)], measured all three contributions $g_{\mathrm{om}}, \kappa_{\mathrm{om}}^{e}$, and $\kappa_{\mathrm{om}}^{i}$ in the $\mathrm{GHz} / \mathrm{nm}$ range [22]. They observed various detuning behaviors depending on the external decay rate (by changing the geometrical characteristics of the input waveguide). Additionally, several coupling configurations have been achieved by Tsvirkun et al., including a situation where the intrinsic dissipative contribution is greater than the other two.

The theoretical framework used to describe optomechanical systems usually considers a purely dispersive interaction (see [1-3]). Dissipative coupling has been discussed in only a few previous works. Elste et al. have assumed that dissipative coupling occurs solely through the modulation of the external optical losses in the particular case of the overcoupled regime $\left(\bar{\kappa}_{e} \gg \bar{\kappa}_{i}\right.$, see erratum of [7]). Weiss et al. have examined an overall dissipative coupling, i.e., a change in the overall optical loss rate with the mechanical motion, in their first study [23]. In their second study, they briefly mentioned the three couplings without discussing the impact of the optical loss regime (see the Appendix of [24]). However, the distinction between the two dissipative couplings and their relative influence in all optical loss regimes has not been clearly considered in these studies.

In this paper, we extend the theoretical framework based on the formalism used to describe purely dispersive optomechanical systems. We give a general description of the optomechanical interactions in the presence of dispersive, external, and intrinsic dissipative couplings, in all optical loss regimes. The proposed extension can be used in various future applications where the distinction between the two 


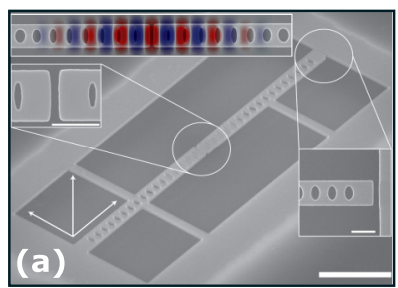

External dissipative $\kappa_{\mathrm{om}}^{e}=\frac{\mathrm{d} \kappa_{e}}{\mathrm{~d} u_{m}}$

$$
\kappa_{e}
$$$$
\kappa_{e}
$$

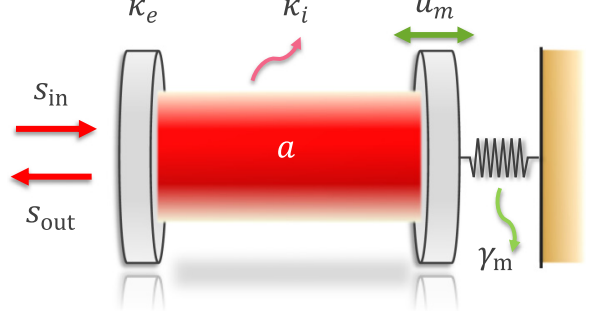

(c)

$$
g_{\mathrm{om}}=\frac{\mathrm{d} \omega_{\mathrm{c}}}{\mathrm{d} u_{m}}
$$

FIG. 1. (a) Scanning electron microscope (SEM) image of a PhC split-beam nanocavity from Wu et al. [20]. (b) SEM image of an integrated $\mathrm{PhC}$ mechanical resonator vertically stacked over a silicon on insulator waveguide from Tsvirkun et al. [22]. (c) Schematic illustration of the equivalent Fabry-Pérot optical cavity with one movable mirror. The important quantities, namely, the normalized intracavity field amplitude $(a)$, the input and output photon flux $\left(s_{\text {in }}\right.$ and $s_{\text {out }}$ ), the external and intrinsic optical decay rates $\left(\kappa_{e}\right.$ and $\left.\kappa_{i}\right)$, the mechanical displacement $\left(u_{m}\right)$, the mechanical intrinsic damping $\left(\gamma_{m}\right)$, the optical resonance frequency $\omega_{c}$, and the three first-order optomechanical couplings $\left(g_{\mathrm{om}}, \kappa_{\mathrm{om}}^{e}\right.$, and $\left.\kappa_{\mathrm{om}}^{i}\right)$, are introduced.

dissipative couplings is necessary. We refer throughout to the $\mathrm{PhC}$ systems of Wu et al. [20] and of Tsvirkun et al. [22] to illustrate our calculations and ascertain their validity in concrete situations.

This paper is organized as follows. In Sec. II, we develop the optical input-output relation and the mechanical dynamical equation in order to establish the general coupled equations of motion. In Sec. III, we analyze the mean output optical response and highlight the influence of each optomechanical contribution on the optical readout of mechanical motion, in all optical loss regimes. This is of practical importance, as it will allow us to experimentally identify the coupling strength of each contribution in future optomechanical systems. In Sec. IV, we derive the impact of each coupling on the intracavity field fluctuations and use the backaction force operator to determine the mechanical spectrum and its associated optomechanical effects (optical spring effect and optomechanical damping). The impact of optical detuning on these quantities is finally compared to the measurements of Tsvirkun et al. [22].

\section{COUPLED EQUATIONS OF MOTION}

In this section we introduce the coupled equations of motion in the presence of the three optomechanical couplings.
They represent the basis of any model aiming to describe the mutual interaction between electromagnetic radiations in an optical cavity and mechanical vibrations. The generic theory developed here is only valid in the linear case, i.e., for small mechanical displacements (first-order perturbations). We consider a generic optomechanical system, constituted by an optical and a mechanical resonator of respective resonance frequencies $\omega_{c}$ and $\omega_{m}$. The optical input-output relation is first used to introduce the three optomechanical couplings. The harmonic oscillator model is then used to describe the mechanical behavior. Finally, we derive the coupled equation of motion by means of a linearization of the optical field and mechanical amplitude around a mean steady-state value.

The optical element is considered to be a one-port cavity in which the light can be coupled in or out by the same side. Typical one-port optical systems are a Fabry-Pérot system with one partially reflective mirror and one perfectly reflective mirror [see Fig. 1(c)] [25], all-pass ring resonators [19], and whispering gallery mode resonators coupled to an optical fiber [18]. The temporal dynamic of the intracavity complex field amplitude $a(t)$ is governed by the input-output relation $[1,26]$

$$
\dot{a}(t)=-\left\{\frac{\kappa\left(u_{m}\right)}{2}-i\left[\omega_{L}-\omega_{c}\left(u_{m}\right)\right]\right\} a(t)+\sqrt{\kappa_{e}\left(u_{m}\right)} s_{\mathrm{in}}(t)
$$

where $u_{m}(t)$ is the temporal mechanical amplitude; $\kappa_{e}$ and $\kappa_{i}$ are, respectively, the external and intrinsic photon decay rates; $\kappa=\kappa_{e}+\kappa_{i}$ is the overall cavity decay rate; $\omega_{L}$ is the input laser angular frequency; and $s_{\text {in }}$ is the input laser flux. The field amplitude is normalized such that $|a|^{2}=n_{\text {cav }}$ stands for the number of intracavity photons. As in most optomechanical systems the optical frequency $\omega_{c}$ depends on the mechanical amplitude $u_{m}$. In our paper, because of the dissipative interactions, we also consider that the decay rates $\kappa_{e}$ and $\kappa_{i}$ depend on $u_{m}$. We thus introduce the dispersive coupling $g_{\text {om }}$, external dissipative coupling $\kappa_{\mathrm{om}}^{e}$, and intrinsic dissipative coupling $\kappa_{\mathrm{om}}^{i}$ which correspond to a shift of, respectively, the cavity resonance frequency, the external decay rate, and the intrinsic decay rate due to the mechanical oscillator motion, which are defined using the first-order Taylor expansion as

$$
\begin{gathered}
\omega_{c}\left(u_{m}\right)=\omega_{c 0}+g_{\mathrm{om}} u_{m}(t), \\
\kappa_{e}\left(u_{m}\right)=\kappa_{e 0}+\kappa_{\mathrm{om}}^{e} u_{m}(t), \\
\kappa_{i}\left(u_{m}\right)=\kappa_{i 0}+\kappa_{\mathrm{om}}^{i} u_{m}(t),
\end{gathered}
$$

where $\omega_{c 0}, \kappa_{e 0}$, and $\kappa_{i 0}$ are, respectively, the optical bare cavity (i.e., without any optomechanical interaction) angular frequency and the external and intrinsic loss rates. It is important to remark that while quadratic coupling can be relevant in some situations, such as in the "membrane-in-the-middle" setup where a purely dispersive quadratic coupling could lead to quantum nondemolition measurement of the mechanical ground state [4], it is beyond the scope of this paper and we will focus only on the effects induced by the three first-order couplings.

The mechanical element is modeled as a harmonic oscillator with intrinsic damping $\gamma_{m}=\omega_{m} / Q_{m}$ (with $Q_{m}$ the mechanical quality factor) and effective mass $m_{\text {eff }}$. The dynamical temporal behavior of the mechanical complex amplitude 
is governed by the equation $[1,27]$

$$
\ddot{u_{m}}(t)+\gamma_{m} \dot{u_{m}}(t)+\omega_{m}^{2} u_{m}(t)=\frac{F_{L}(t)}{m_{\mathrm{eff}}}+\frac{F_{\mathrm{opt}}(t)}{m_{\mathrm{eff}}},
$$

where $F_{L}(t)$ represents the thermal Langevin force arising from the thermal fluctuations and responsible for the Brownian motion of the mechanical resonator, and $F_{\mathrm{opt}}(t)$ is the optical force induced by the intracavity field.

In order to derive the coupled optomechanical equations of motion, we expand and linearize the input-output relation for the intracavity field. Consider the mean steady-state value of the field amplitude $\bar{a}$ and mechanical displacement $\bar{u}_{m}$ and their respective temporal fluctuations $\delta a(t)$ and $\delta u_{m}(t)$ such that $a(t)=[\bar{a}+\delta a(t)] e^{-i \omega_{L} t}$ and $u_{m}(t)=\bar{u}_{m}+\delta u_{m}(t)$. Second-order terms such as $\delta a(t) \delta u_{m}(t)$ are neglected. We consider a continuous input flux $s_{\text {in }}=\bar{s}_{\text {in }} e^{-i \omega_{L} t}$ with $\bar{s}_{\text {in }}$ constant. We define (1) $\bar{\Delta}=\Delta_{0}-g_{\text {om }} \bar{u}_{m}$ the effective optical detuning (with $\Delta_{0}=\omega_{L}-\omega_{c 0}$ ), (2) $\bar{\kappa}_{e 0}=\kappa_{e 0}+\kappa_{\mathrm{om}}^{e} \bar{u}_{m}$ the effective external photon decay rate, (3) $\bar{\kappa}_{i 0}=\kappa_{i 0}+\kappa_{\mathrm{om}}^{i} \bar{u}_{m}$ the effective intrinsic photon decay rate, and (4) $\bar{\kappa}=\bar{\kappa}_{e 0}+\bar{\kappa}_{i 0}$ the effective overall photon decay rate.

Under the assumption of small mechanical displacement fluctuations, we can use the first-order expansion

$$
\sqrt{\kappa_{e}\left(u_{m}\right)}=\sqrt{\bar{\kappa}_{e 0}}\left(1+\frac{\kappa_{\mathrm{om}}^{e}}{2 \bar{\kappa}_{e 0}} \delta u_{m}(t)\right)
$$

in the optical input-output relation (1). We linearize Eqs. (1) and (5) by means of all the definitions previously introduced to obtain Eqs. (7)-(10). The mean intracavity field and displacement amplitudes are given by

$$
\begin{array}{r}
\bar{a}=\frac{\sqrt{\bar{\kappa}_{e 0}}}{\bar{\kappa} / 2-i \bar{\Delta}} \bar{s}_{\mathrm{in}}, \\
\bar{u}_{m}=\frac{\bar{F}_{\mathrm{opt}}}{m_{\mathrm{eff}} \omega_{m}^{2}},
\end{array}
$$

where $\bar{F}_{\text {opt }}$ represents the mean optical force responsible for the change in the detuning and the decay rates. Note that a nonlinear effect named static bistability arises because of the impact of the mean mechanical displacement on the optical parameters: under certain conditions, Eqs. (7) and (8) have multiple solutions, giving rise to several stable behaviors, which have been studied in dispersive and dissipative optomechanical systems [28-30].

In addition, the field and displacement fluctuations obey the following differential equations:

$$
\begin{aligned}
& \dot{\delta a}(t)=\left(-\frac{\bar{\kappa}}{2}+i \bar{\Delta}\right) \delta a(t)-\left[\left(\frac{\kappa_{\mathrm{om}}^{e}+\kappa_{\mathrm{om}}^{i}}{2}+i g_{\mathrm{om}}\right) \bar{a}\right. \\
&\left.-\frac{\kappa_{\mathrm{om}}^{e}}{2 \sqrt{\bar{\kappa}_{e 0}}} \bar{s}_{\mathrm{in}}\right] \delta u_{m}(t)+\sqrt{\bar{\kappa}_{e 0}} \bar{s}_{\mathrm{in}}, \\
& \delta \ddot{u}_{m}(t)+\gamma_{m} \delta \dot{u}_{m}(t)+\omega_{m}^{2} \delta u_{m}(t)=\frac{\delta F_{L}(t)}{m_{\mathrm{eff}}}+\frac{\delta F_{\mathrm{opt}}(t)}{m_{\mathrm{eff}}} .
\end{aligned}
$$

While the purely dispersive terms remain unchanged [1-3], we can observe that the dissipative couplings give rise to supplementary terms proportional to the mechanical displacement fluctuations. As observed in a previous study on dissipative optomechanical systems [23], and in contrast to the dispersive case which is a purely cavity assisted coupling proportional to the intracavity steady-state field $\bar{a}$, the dissipative terms are also proportional to the drive flux $s_{\text {in. }}$. In our paper, because we distinguish the intrinsic and external components, we trace this effect back to the external dissipative coupling.

In our model, the optical cavity is assumed to be a one-port system. For multiport optical systems, the model can still be valid by defining different optical loss rates. We also did not consider any external modulation of the input field, which may add additional terms to the previous equations.

In the following, the influence of each coupling on the mean optical response is studied. The two coupled equations of motion (9) and (10) are then used as a starting point to derive the mechanical spectrum and the optomechanical effects in the presence of dispersive, external, and intrinsic dissipative couplings.

\section{OUTPUT OPTICAL RESPONSE}

In order to fully understand the basic optical behavior of an optomechanical system with the three couplings, the mean steady-state output optical response is studied. The optical loss regimes are defined and their influence on the mechanically induced optical power oscillations is analyzed. To this end, the mean output optical response has to be determined. As we are considering a one-port optical cavity, there is a single output photon flux $s_{\text {out }}$ [see (1)]. The input-output relation for this output flux is given by

$$
s_{\text {out }}(t)=\sqrt{\kappa_{e}\left(u_{m}\right)} a(t)-\bar{s}_{\text {in }} .
$$

After linearization of this relation, the mean steady-state value of the optical flux $\bar{s}_{\text {out }}$ is deduced by means of (7):

$$
\bar{s}_{\text {out }}=\frac{\bar{\kappa}_{e 0}-\bar{\kappa} / 2+i \bar{\Delta}}{\bar{\kappa} / 2-i \bar{\Delta}} \bar{s}_{\text {in }} .
$$

The mean steady-state output response, defined as $R_{\text {out }}=$ $\left|\bar{s}_{\text {out }} / \bar{s}_{\text {in }}\right|^{2}$, reads

$$
R_{\text {out }}=\frac{\left(\bar{\kappa}_{e 0}-\bar{\kappa} / 2\right)^{2}+\bar{\Delta}^{2}}{(\bar{\kappa} / 2)^{2}+\bar{\Delta}^{2}} .
$$

Practically speaking, $R_{\text {out }}$ is similar to the typical reflection response of a one-port Fabry-Pérot cavity (with one mirror partially and the other one perfectly reflective). The mechanically induced optical oscillations are represented by $d R_{\text {out }} / d u_{m}$, which is given by

$$
\frac{d R_{\text {out }}}{d u_{m}}=g_{\text {om }} \frac{\partial R_{\text {out }}}{\partial \bar{\Delta}}+\kappa_{\mathrm{om}}^{e} \frac{\partial R_{\text {out }}}{\partial \bar{\kappa}_{e}}+\kappa_{\text {om }}^{i} \frac{\partial R_{\text {out }}}{\partial \bar{\kappa}_{i}},
$$

where the derivatives of $R_{\text {out }}$ are given by

$$
\begin{gathered}
\frac{\partial R_{\text {out }}}{\partial \bar{\Delta}}=\frac{2 \bar{\Delta}\left(1-R_{\text {out }}\right)}{(\bar{\kappa} / 2)^{2}+\bar{\Delta}^{2}}, \\
\frac{\partial R_{\text {out }}}{\partial \bar{\kappa}_{e}}=\frac{\left(\bar{\kappa} / 2-\bar{\kappa}_{e 0}\right)-(\bar{\kappa} / 2) R_{\text {out }}}{(\bar{\kappa} / 2)^{2}+\bar{\Delta}^{2}}, \\
\frac{\partial R_{\text {out }}}{\partial \bar{\kappa}_{i}}=\frac{\left(\bar{\kappa}_{e 0}-\bar{\kappa} / 2\right)-(\bar{\kappa} / 2) R_{\text {out }}}{(\bar{\kappa} / 2)^{2}+\bar{\Delta}^{2}} .
\end{gathered}
$$

In the following sections, we first present a qualitative analysis of each derivative term under different values of the external decay rate $\bar{\kappa}_{e 0}$, and identify which optomechanical 

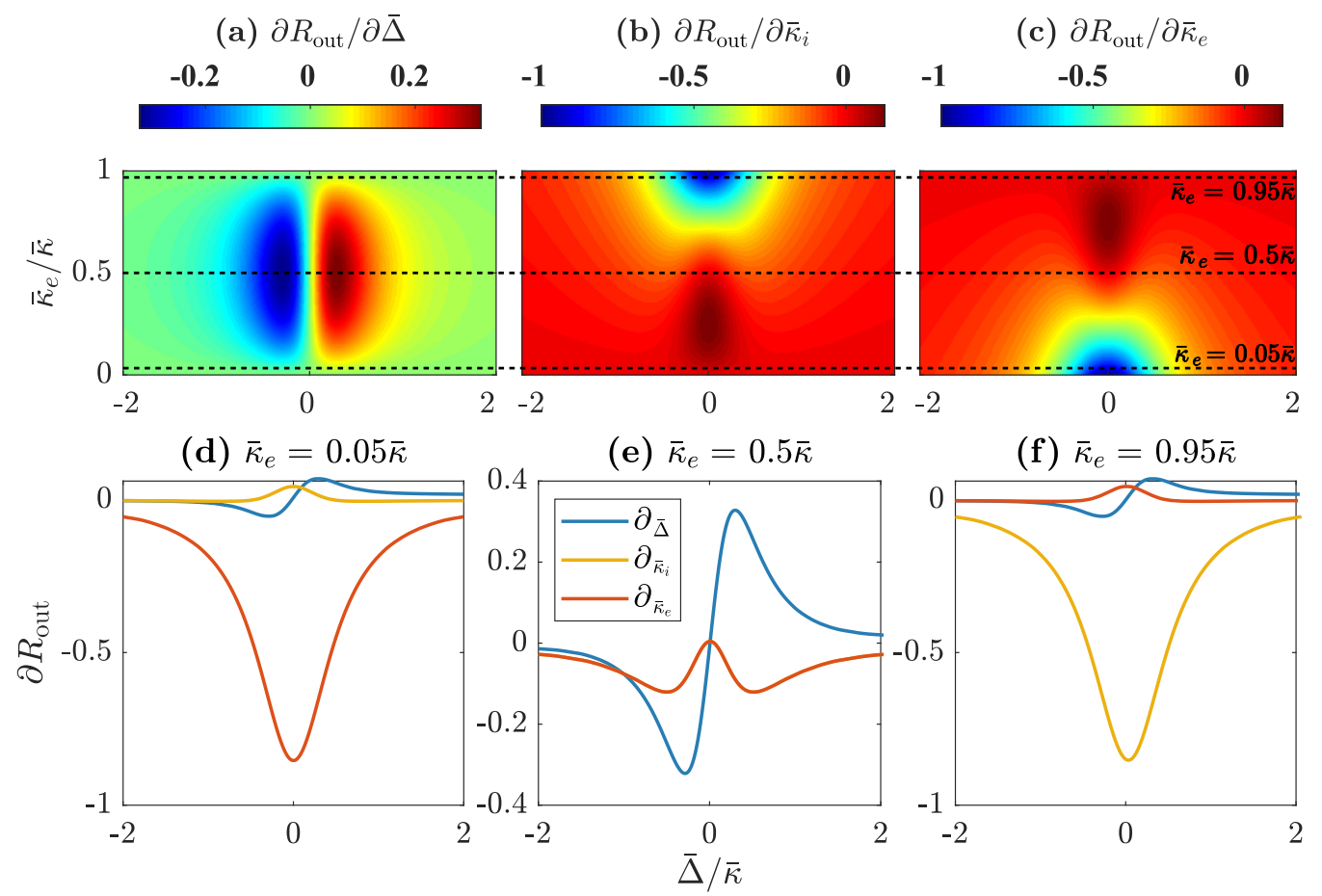

FIG. 2. Derivatives of the mean optical response amplitude as a function of the normalized detuning $\bar{\Delta} / \bar{\kappa}$. Influence of the normalized external cavity decay rate $\bar{\kappa}_{e 0} / \bar{\kappa}$ on (a) $\partial R_{\text {out }} / \partial \bar{\Delta}$ (blue curve), (b) $\partial R_{\text {out }} / \partial \bar{\kappa}_{i 0}$ (yellow curve), and (c) $\partial R_{\text {out }} / \partial \bar{\kappa}_{e 0}($ red curve). Comparison between the three derivative amplitudes in the (d) undercoupled regime, (e) critically coupled regime (yellow and red curves are overlapping), and (f) overcoupled regime. For the six plots, the amplitudes are normalized with the maximum value between the three derivatives. The comparison made here is purely qualitative and is independent of the value of $\bar{\kappa}$.

interaction (namely, dispersive, intrinsic, and external) is enhanced depending on the considered optical loss regime. We then conduct the analysis with a more quantitative approach, with different values of the optomechanical coupling rates $g_{\mathrm{om}}, \kappa_{\mathrm{om}}^{e}$, and $\kappa_{\mathrm{om}}^{i}$ to understand their relative impact on the amplitude of the mechanically induced mean optical power oscillations.

\section{A. Influence of the optical loss regime}

The variations of the mean optical response, given by the three derivatives previously calculated, with the three quantities of interest, namely, the optical detuning $\bar{\Delta}$, the external loss rate $\bar{\kappa}_{e}$, and the intrinsic loss rate $\bar{\kappa}_{i}$, allow us to identify the optical loss regimes. They are displayed as a function of the detuning $\bar{\Delta}$ and the external decay rate $\bar{\kappa}_{e}$ in Fig. 2. All the plots have been normalized with the maximum value between $\partial R_{\text {out }} / \partial \bar{\Delta}, \partial R_{\text {out }} / \partial \bar{\kappa}_{e}$, and $\partial R_{\text {out }} / \partial \bar{\kappa}_{i}$, and the detuning $\bar{\Delta}$ and external decay rate $\bar{\kappa}_{e 0}$ have been normalized with the overall decay rate $\bar{\kappa}$, such that the previous analysis is general and independent of the quality of the optical cavity, i.e., independent of $\bar{\kappa}$. The influence of the external cavity decay rate $\bar{\kappa}_{e 0}$ is shown in Figs. 2(a)-2(c), highlighting the most important discrepancy between the dispersive and dissipative derivatives: the dispersive behavior is characterized by two off resonant sidebands whereas the dissipative (both intrinsic and external) behavior is characterized by a single resonant maximum. We can thus identify three particular optical loss regimes and compare the detuning dependency of the three derivatives within each of them: (1) the undercoupled regime, $\bar{\kappa}_{e 0} \ll \bar{\kappa} \approx \bar{\kappa}_{i 0}$ [Fig. 2(d)]; (2) the critically coupled regime, $\bar{\kappa}_{e 0}=\bar{\kappa} / 2=\bar{\kappa}_{i 0}$ [Fig. 2(e)]; and (3) the overcoupled regime, $\bar{\kappa}_{e 0} \approx \bar{\kappa} \gg \bar{\kappa}_{i 0}$ [Fig. 2(f)].

In the critically coupled regime [see Fig. 2(e)], the dispersive variation (blue curve) of the mean optical response is the predominant mechanism, with the highest impact at off resonance detunings. It confirms the interest of this regime in purely dispersive optomechanical systems in which this interaction is strongly enhanced. It also justifies that the two dissipative optomechanical couplings are not considered in the common theoretical model. However, since the dissipative contribution is not negligible, we will see in the next section that it also induces various effects on the mean optical power oscillations.

In the undercoupled regime [see Fig. 2(d)], intrinsic losses $\left(\bar{\kappa}_{i 0}\right)$ are higher and the external dissipative variation (red curve) is the predominant mechanism. As a result, on resonance $(\bar{\Delta}=0)$, a small variation of the intrinsic cavity decay rate $\bar{\kappa}_{i}$ will not have a strong influence on the optical output response $R_{\text {out }}$, in contrast to a small variation of external cavity decay rate $\bar{\kappa}_{e}$ which can have a significant impact. In [20], the system is in this undercoupled regime $(\bar{\kappa}=31 \mathrm{GHz}$ and $\bar{\kappa}_{e 0}=1 \mathrm{GHz}$, i.e., $\bar{\kappa}_{e 0}=0.03 \bar{\kappa}$ ), and the authors indeed observed a strong dependence of the mean optical response on the external decay rate.

In the overcoupled regime, the detuning dependency of the intrinsic (respectively, external) dissipative variations is identical to the detuning dependency of the external (respectively, intrinsic) dissipative variations in the undercoupled regime, due to the mathematical symmetry between Eqs. (16) and 


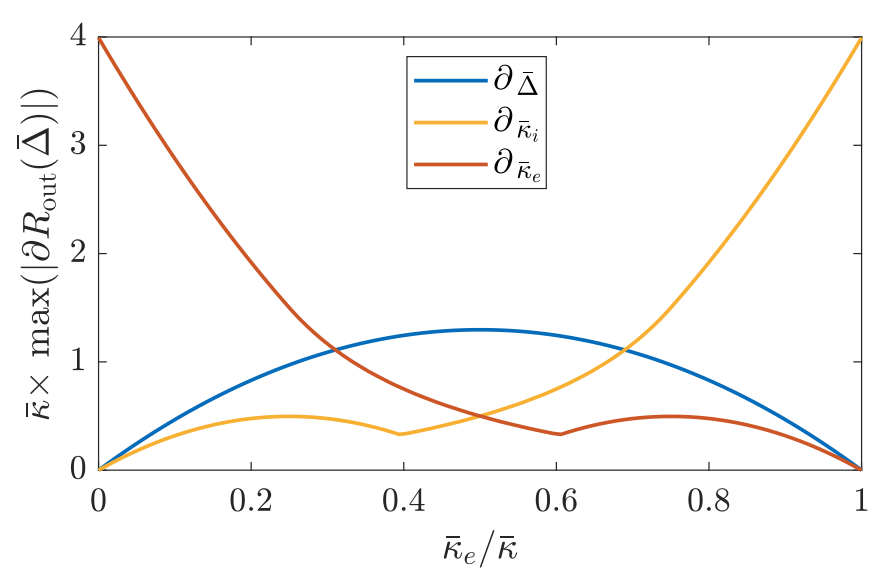

FIG. 3. Absolute maximum of the derivatives of the mean optical response as a function of the normalized external cavity decay rate $\bar{\kappa}_{e} / \bar{\kappa}$. The $y$ axis is normalized such that $y$ values are independent of $\bar{\kappa}$.

(17). Note that for the last two regimes the dispersive variation is negligible.

The dispersive derivative of the mean optical response $\left(\partial R_{\text {out }} / \partial \bar{\Delta}\right)$ is always an off resonance effect associated with two sidebands. These sidebands correspond to dynamical effects (cooling or amplification of the mechanical oscillations [1-3]). Conversely the maximum of each dissipative derivative $\left(\partial R_{\text {out }} / \partial \bar{\kappa}_{e}\right.$ and $\left.\partial R_{\text {out }} / \partial \bar{\kappa}_{i}\right)$ always occurs on resonance. However, depending on the optical loss regime, two off resonance local maxima appear [close to the critically coupled regime, see red and yellow curves in Fig. 2(e)], giving rise to new working sidebands. These are related to new optical detunings for which cooling or amplification of the mechanical motion can occur [23]. Figure 3 represents the maximum amplitude of the derivatives of $R_{\text {out }}$ as a function of the external cavity decay rate. These maximum amplitudes are given by

$$
\begin{gathered}
\left.\frac{\partial R_{\text {out }}}{\partial \bar{\Delta}}\right|_{\max }=\left|\frac{2\left[1-R_{\text {out }}\left(\bar{\Delta}= \pm \bar{\Delta}_{\text {max }}\right)\right]}{\bar{\kappa}}\right| \\
\left.\frac{\partial R_{\text {out }}}{\partial \bar{\kappa}_{e}}\right|_{\max }=\left|\frac{\left(\bar{\kappa} / 2-\bar{\kappa}_{e 0}\right)-(\bar{\kappa} / 2) R_{\text {out }}(\bar{\Delta}=0)}{=\frac{3 \sqrt{3}}{4 \bar{\kappa}},}\right| \\
\left.\frac{\partial R_{\text {out }}}{\partial \bar{\kappa}_{i}}\right|_{\max }=\mid \frac{(\bar{\kappa} / 2)^{2}}{=} \frac{4}{\bar{\kappa}}, \\
\left.\bar{\kappa}_{e 0}-\bar{\kappa} / 2\right)-(\bar{\kappa} / 2) R_{\text {out }}(\bar{\Delta}=0) \\
\stackrel{(\bar{\kappa} / 2)^{2}}{=} \frac{4}{\bar{\kappa}},
\end{gathered}
$$

where $\bar{\Delta}_{\max }=\bar{\kappa} / \sqrt{12}$ is the detuning of maximum slope of the mean optical response, which is determined by making $\partial^{2} R_{\text {out }} / \partial \bar{\Delta}^{2}$ equal to zero. The maximum of each variation of the mean optical response is consequently inversely proportional to the overall cavity decay rate $\bar{\kappa}$, which highlights the interest of working in the resolved sideband regime $\left(\bar{\kappa} \ll \omega_{m}\right)$ to increase optical sensibility towards mechanical
TABLE I. Optomechanical coupling absolute values and sideband factor measured by Wu et al. [20] and Tsvirkun et al. [22].

\begin{tabular}{lcc}
\hline \hline & Wu et al. [20] & Tsvirkun et al. $[22]$ \\
\hline$g_{\text {om }}(\mathrm{GHz} / \mathrm{nm})$ & $1.1-1.8$ & $0.62-1.52$ \\
$\kappa_{\mathrm{om}}^{e}(\mathrm{GHz} / \mathrm{nm})$ & $0.002-0.003$ & $0.13-0.33$ \\
$\kappa_{\mathrm{om}}^{i}(\mathrm{GHz} / \mathrm{nm})$ & $0.3-0.5$ & $0.01-5.64$ \\
$\bar{\kappa} / \omega_{m}$ & $10^{3}$ & $10^{3}-10^{4}$ \\
\hline \hline
\end{tabular}

displacement. We see that no matter the value of the optical cavity decay rate the dissipative variations (both external and intrinsic) of the output response in the corresponding optical loss regime (respectively, undercoupled and overcoupled) are always more than three times higher $(16 / 3 \sqrt{3} \approx 3.1)$ than the dispersive one in the critically coupled regime. It emphasizes the interest of dissipative coupling optical readout of the mechanical motion (for classical applications), in the corresponding optical loss regime.

Our paper helps to clearly identify these three regimes. The plots of Fig. 2 qualitatively summarize the steady-state optical behavior, common to all optomechanical systems with the three couplings. In practice, however, we measure the optical power and have access to the total variation of the optical power response. It is at this point that a quantitative study, taking into account the coupling values $g_{\mathrm{om}}, \kappa_{\mathrm{om}}^{e}$, and $\kappa_{\mathrm{om}}^{i}$, is necessary.

\section{B. Mean optical power oscillations}

The dependence of the mean optical power oscillations of our generic system on the optical detuning and external cavity decay rate is thus quantitatively studied based on different optomechanical coupling configurations. To this end, we define the output power as $P_{\text {out }}=R_{\text {out }} P_{\text {in }}$ with $P_{\text {in }}$ the input power. Based on Eq. (14), the total derivative of the optical response is the sum of each partial derivative weighted by the optomechanical coupling values. Therefore the mechanically induced mean optical power oscillations are given by $d P_{\text {out }} / d u_{m}=P_{\text {in }} d R_{\text {out }} / d u_{m}$ in $\mathrm{W} / \mathrm{m}$. Note that we actually use an input power of $1 \mathrm{~mW}$ and thus the $\mu \mathrm{W} / \mathrm{pm}$ unit of measurement for convenience.

Table I summarizes the typical absolute coupling values and corresponding sideband parameter $\bar{\kappa} / \omega_{m}$ measured by Wu and coworkers in one of their $\mathrm{PhC}$ split-beam nanocavities $[20,21]$ and by Tsvirkun et al. in their PhC slab suspended over an input waveguide [22,31]. The best optomechanical systems are designed for dispersive optomechanical cooling applications in the resolved sideband regime and achieved $\bar{\kappa}=0.02 \omega_{m}[1]$. However, based on both the measurements of $\mathrm{Wu}$ and coworkers [20,21] and Tsvirkun et al. [22], we notice that the sideband factor $\bar{\kappa} / \omega_{m}$ has no impact on the achievable dispersive and dissipative coupling values. The amplitude of mechanically induced mean optical power oscillations is not influenced by this parameter, but only by the value of $\bar{\kappa}$ itself, i.e., by the quality of the optical cavity. It is also independent of the mechanical resonator properties (resonant frequency $\omega_{m}$, quality factor $Q_{m}$, and effective mass $\left.m_{\text {eff }}\right)$. However, the sideband regime influences the optically induced effects on the mechanical properties (see last section). 


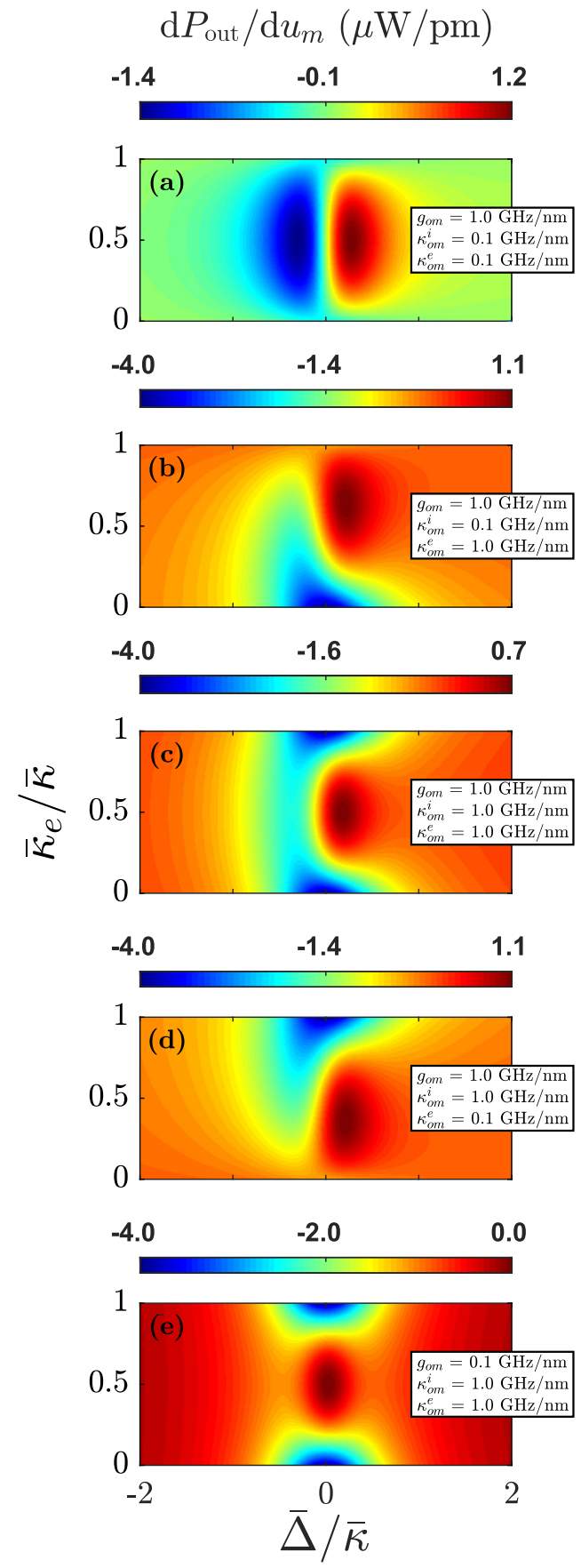

FIG. 4. Mechanically induced mean optical power oscillations as a function of the normalized detuning $\bar{\Delta} / \bar{\kappa}$ and the normalized external cavity decay rate $\bar{\kappa}_{e} / \bar{\kappa}$ for different optomechanical coupling configurations, in the unresolved sideband limit $\bar{\kappa}=10^{3} \omega_{m}$ with $\omega_{m}=1 \mathrm{MHz}$.

Figure 4 represents the mechanically induced mean optical power oscillations $d P_{\text {out }} / d u_{m}$ as a function of the optical detuning and the external loss rate for five optomechanical coupling configurations (the coupling values are given in the figure). These are related to five different coupling regimes with strong physical behavior discrepancies both on the mean optical behavior and on the dynamical effects that we will analyze in the last section. We set $\bar{\kappa}=10^{3} \omega_{m}=$
TABLE II. Mechanical and optical parameters used to describe the $\mathrm{PhC}$ devices of Tsvirkun et al. All parameters have been retrieved from [22,31] (mechanical mode labeled M1), except for $\bar{\kappa}$ and $\bar{\kappa}_{e}$, which have been deduced for each configuration.

\begin{tabular}{lcc}
\hline \hline \multicolumn{3}{c}{ Mechanical properties } \\
\hline$\omega_{m}$ & \multicolumn{3}{c}{$2.22 \mathrm{MHz}$} \\
$Q_{m}$ & 2000 \\
$m_{\text {eff }}$ & \multicolumn{3}{c}{ Optical properties } \\
\hline \multicolumn{3}{c}{$6.8 \mathrm{~mW}$} \\
$P_{\text {in }}$ & \multicolumn{3}{c}{$1563.42 \mathrm{~nm}$} \\
$\lambda_{\text {cav }}$ & $0.09 \mathrm{~nm}$, i.e., $11 \mathrm{GHz}$ & (Fig. 5) \\
$\bar{\kappa}$ & $0.18 \mathrm{~nm}$, i.e., $22 \mathrm{GHz}$ & [Figs. 6(a) and 6(b)] \\
\multicolumn{4}{c}{ [Figs. 6(c) and 6(d)] } \\
$\bar{\kappa}_{e}$ & $0.55 \mathrm{~nm}$, i.e., 190 GHz & [Figs. 6(e) and 6(f)] \\
& $0.16 \bar{\kappa}$ & [Figs. 6(a) and 6(b)] \\
& $0.08 \bar{\kappa}$ & [Figs. 6(c) and 6(d)] \\
& $0.21 \bar{\kappa}$ & [Figs. 6(e) and 6(f)] \\
\hline \hline
\end{tabular}

$1 \mathrm{GHz}$ (based on one of the devices of Tsvirkun et al. [22,31]). Figure 4 shows that in this case the maximum absolute power oscillations are around $4 \mu \mathrm{W} / \mathrm{pm}$, for $g_{\text {om }}$, $\kappa_{\mathrm{om}}^{e}$, and $\kappa_{\mathrm{om}}^{i}$ at $1 \mathrm{GHz} / \mathrm{nm}$, and that this value increases linearly when decreasing $\bar{\kappa}\left(0.4 \mathrm{~mW} / \mathrm{pm}\right.$ for $\bar{\kappa}=10, \omega_{m}=$ $10 \mathrm{MHz}$ ). In the absence of optomechanical effects, we can easily calculate the corresponding thermal optical spectrum. For instance, consider a mechanical resonator with $m_{\text {eff }}=$ $117.2 \mathrm{pg}$ and $Q_{m}=2000$ (from Tsvirkun et al., see Table II). The associated resonant Brownian motion at room temperature $(T=294 \mathrm{~K})$ is given by $\sqrt{S_{\mathrm{th}}}=\sqrt{4 k_{B} T Q_{m} / m_{\mathrm{eff}} \omega_{m}^{3}}$ [27] where $k_{B}=1.38 \times 10^{-23} \mathrm{~m}^{2} \mathrm{~kg} / \mathrm{s}^{2} \mathrm{~K}$ is the Boltzmann constant. Thus we have $\sqrt{S_{\mathrm{th}}} \sim 0.16 \mathrm{pm} / \sqrt{\mathrm{Hz}}$, and the associated maximum optical power spectral density (PSD) is $\sqrt{S_{\text {opt,th }}} \sim$ $0.6 \mu \mathrm{W} / \sqrt{\mathrm{Hz}}$ for $\bar{\kappa}=1 \mathrm{GHz}$ (and $\sqrt{S_{\mathrm{opt}, \mathrm{th}}} \sim 60 \mu \mathrm{W} / \sqrt{\mathrm{Hz}}$ for $\bar{\kappa}=10 \mathrm{MHz}$ ). As a remark, we point out that the previous calculation is based on values from Tsvirkun et al. [22,31], which, although typical, may vary according to the relative strength of the optomechanical couplings. Nevertheless, the calculation remains relevant because it gives an order of magnitude of the quantity effectively measured.

Figure 4(a) illustrates a mostly dispersive case where the strongest mean optical power oscillations arise at critical coupling and off resonant detuning. We consider relatively low dissipative couplings in comparison to $g_{\text {om }}$, which explains the low power oscillations in the undercoupled and overcoupled regime. However, we observe an asymmetry in the amplitude of the dispersive sidebands due to the nonzero dissipative couplings. Indeed, the comparison with Fig. 2 reveals that the dissipative mechanisms induce, on resonance, a negative (or positive, depending of the sign of the couplings) amplification of the power oscillations which are added to or subtracted from the dispersive sidebands. This effect is responsible for the strong asymmetry of the five plots. Figures 4(b) and 4(d) show the influence of a stronger, respectively, external and intrinsic dissipative coupling, with the same dispersive coupling value. We observe an amplification of the asymmetry 
in the critically coupled regime. The highest power oscillations in these two cases are achievable on resonance in the undercoupled (for higher external dissipative coupling) or overcoupled (for higher intrinsic dissipative coupling) regime. Figure 4(c) illustrates the case where the three optomechanical couplings are at the same level (Tsvirkun et al. were close to this situation in one of their devices, see Fig. 3(d) in [22]). Finally, Fig. 4(e) shows the dissipative case in which dispersive optomechanical coupling is negligible in comparison to the two other couplings, almost completely extinguishing the dispersive detuning sidebands in the critically coupled regime. In the last two situations, working in the undercoupled or overcoupled regime will induce the same strong optical power oscillations on optical resonance. The sign of each coupling can also induce other discrepancies, but the behavior does not drastically change. The absolute mean optical power oscillations remain in the same order of magnitude, but maximum absolute values can arise at different detuning values in the critically coupled regime.

The five plots of Fig. 4 summarize all the physical situations it is possible to encounter when measuring the mean optical response of an optomechanical setup. Our analysis reveals that the optical loss regime and the optomechanical coupling configuration have a strong influence on the optical behavior. As pointed out in [20], the tools introduced here can be used to identify the magnitudes of each coupling in practical optomechanical experiments. To achieve this, one must fit the optical power oscillations at mechanical resonance by means of Eqs. (14)-(17) to estimate the relative contribution (i.e., the coupling strengths $g_{\mathrm{om}}, \kappa_{\mathrm{om}}^{i}$, and $\kappa_{\mathrm{om}}^{i}$ ) of each optomechanical coupling process. These tools have been used by Wu et al. [20] and Tsvirkun et al. [22] in their own systems, the observed detuning behaviors of which correspond to specific cases in our analysis. As this basic model uses the general expression of the mean output optical response, it is very general and can thus be applied to a large variety of systems. However, to fully understand the physical phenomena induced by the three types of coupling in an optomechanical device, we need to analyze the mechanical dynamic and determine the optically induced effects on the mechanical properties.

\section{MECHANICAL SPECTRUM}

In the following, we investigate the dynamical properties of the mechanical resonator. We first use the input-output relation [see Eq. (9)] in order to obtain the Fourier transform of the intracavity field fluctuations. After calculating the optical force in the presence of dispersive and dissipative couplings, the mechanical response is determined, from which the general expressions of the optical spring effect and optomechanical damping are extracted. Finally, the theoretical optical spectrum in a concrete case is compared with previous measurements from Tsvirkun et al. [22].

From now on, we work in the Fourier space and choose the convention $a(\omega)=\int_{-\infty}^{+\infty} a(t) e^{-i \omega t}$. The Fourier transform of Eq. (9) allows us to write the fluctuations of the intracavity field as

$$
\delta a(\omega)=\delta a_{\mathrm{disp}}(\omega)+\delta a_{\mathrm{diss}}^{e}(\omega)+\delta a_{\mathrm{diss}}^{i}(\omega),
$$

with $\delta a_{\text {disp }}(\omega), \delta a_{\text {diss }}^{e}(\omega)$, and $\delta a_{\text {diss }}^{i}(\omega)$ the fluctuations induced by, respectively, the dispersive, the external dissipative, and the intrinsic dissipative coupling given by

$$
\begin{gathered}
\delta a_{\mathrm{disp}}(\omega)=-i g_{\mathrm{om}} \chi_{\mathrm{cav}}^{\mathrm{eff}}(\omega) \bar{a} \delta u_{m}(\omega), \\
\delta a_{\mathrm{diss}}^{e}(\omega)=\frac{\kappa_{\mathrm{om}}^{e}\left(\bar{\kappa} / 2-\bar{\kappa}_{e 0}-i \bar{\Delta}\right)}{2 \bar{\kappa}_{e 0}} \chi_{\mathrm{cav}}^{\mathrm{eff}}(\omega) \bar{a} \delta u_{m}(\omega), \\
\delta a_{\mathrm{diss}}^{i}(\omega)=-\frac{\kappa_{\mathrm{om}}^{i}}{2} \chi_{\mathrm{cav}}^{\mathrm{eff}}(\omega) \bar{a} \delta u_{m}(\omega) .
\end{gathered}
$$

Here we recognize in each term the effective cavity response in the presence of optomechanical interaction $\chi_{\text {cav }}^{\text {eff }}(\omega)=[\bar{\kappa} / 2-i(\bar{\Delta}+\omega)]^{-1}$ which is due to the filtering role of the resonant optical cavity [1]. The three couplings lead to an optical force $F_{\text {opt }}(t)$ the fluctuations of which can be described by the backaction force operator [7,15], which in the case of a constant input flux [i.e., $\bar{s}_{\text {in }}(t)=\bar{s}_{\text {in }}$ ] is written

$$
\begin{aligned}
\delta F_{\mathrm{opt}}(t)= & -\hbar g_{\mathrm{om}}\left[\bar{a}^{*} \delta a(t)+\bar{a} \delta a^{*}(t)\right] \\
& -i \hbar \frac{\kappa_{\mathrm{om}}^{e}+\kappa_{\mathrm{om}}^{i}}{2} \frac{s_{\mathrm{in}}}{\sqrt{\bar{\kappa}_{e 0}}}\left[\delta a^{*}(t)-\delta a(t)\right] .
\end{aligned}
$$

The first term corresponds to the dispersive optical force and is linked to the intracavity photon energy, which varies with mechanical displacement. The second term is the dissipative optical force, analogous to a viscous force and originating from the photons leaking out of the cavity via external or intrinsic dissipation mechanisms. This general expression leads to a linear relation between the Fourier transforms of the optical force and displacement fluctuations. The effective mechanical susceptibility $\chi_{m}^{\text {eff }}$ defined by $\delta u_{m}(\omega, \bar{\Delta})=$ $\chi_{m}^{\text {eff }}(\omega, \bar{\Delta}) F_{L}(\omega)$ is thus determined by

$$
\chi_{m}^{\mathrm{eff}}(\omega, \bar{\Delta})=\frac{1}{m_{\mathrm{eff}}\left(\omega_{m}^{2}-\omega^{2}-i \omega \gamma_{m}\right)+\Sigma(\omega, \bar{\Delta})}
$$

where

$$
\Sigma(\omega, \bar{\Delta})=-\frac{\delta F_{\mathrm{opt}}}{\delta u_{m}}
$$

is the optomechanical self-energy [1]. We can introduce the optical spring effect $\delta \omega_{m}$ and optomechanical damping $\gamma_{\text {opt }}$ with the relation $\Sigma=m_{\mathrm{eff}}\left(2 \omega \delta \omega_{m}-i \omega \gamma_{\mathrm{opt}}\right)$. These two quantities are then expressed as

$$
\begin{gathered}
\delta \omega_{m}(\omega, \bar{\Delta})=-\frac{1}{2 \omega m_{\mathrm{eff}}} \operatorname{Re}\left[\frac{\delta F_{\mathrm{opt}}}{\delta u_{m}}\right], \\
\gamma_{\mathrm{opt}}(\omega, \bar{\Delta})=\frac{1}{\omega m_{\mathrm{eff}}} \operatorname{Im}\left[\frac{\delta F_{\mathrm{opt}}}{\delta u_{m}}\right],
\end{gathered}
$$

where Re and Im, respectively, stand for real and imaginary parts. Because the optical force is composed of three forces induced by each coupling, the optically induced effects are composed of three terms proportional to $g_{\text {om }}^{2},\left(\kappa_{\mathrm{om}}^{e}\right)^{2}$, and $\left(\kappa_{\mathrm{om}}^{i}\right)^{2}$ corresponding, respectively, to the purely dispersive, external dissipative, and intrinsic dissipative situation. However, as the intracavity field fluctuations depend on the three couplings at the same time [see Eqs. (22)-(24)], there are also "interferences" between them which lead to intertwined 
terms proportional to $g_{\mathrm{om}} \kappa_{\mathrm{om}}^{e}, g_{\mathrm{om}} \kappa_{\mathrm{om}}^{i}$, and $\kappa_{\mathrm{om}}^{e} \kappa_{\mathrm{om}}^{i}$ (see the Appendix).

The sum of the contributions of the purely dispersive, external and intrinsic dissipative, and crossing terms leads to the overall optical spring effect and optomechanical damping in the presence of the three couplings:

$$
\begin{aligned}
\delta \omega_{m}=\delta & \omega_{m}^{\mathrm{disp}}+\delta \omega_{m}^{\mathrm{diss}, e}(\omega)+\delta \omega_{m}^{\mathrm{diss}, i} \\
+ & \delta \omega_{m}^{\mathrm{disp}, \mathrm{diss}, e}+\delta \omega_{m}^{\mathrm{disp}, \mathrm{diss}, i}+\delta \omega_{m}^{\mathrm{diss}, e, \text { diss }, i} \\
\gamma_{\mathrm{opt}}= & \gamma_{\mathrm{opt}}^{\mathrm{disp}}+\gamma_{\mathrm{opt}}^{\mathrm{diss}, e}+\gamma_{\mathrm{opt}}^{\mathrm{diss}, i}+\gamma_{\mathrm{opt}}^{\mathrm{disp}, \mathrm{diss}, e} \\
& +\gamma_{\mathrm{opt}}^{\mathrm{disp}, \mathrm{diss}, i}+\gamma_{\mathrm{opt}}^{\mathrm{diss}, e \text { diss }, i} .
\end{aligned}
$$

In practical experiments, we have access to the optical PSD $S_{\text {opt }}(\omega, \bar{\Delta})$ in $\mathrm{W}^{2} / \mathrm{Hz}$, related to the mechanical PSD $S_{m}(\omega, \vec{\Delta})$ in $\mathrm{m}^{2} / \mathrm{Hz}$ by

$$
S_{\text {opt }}(\omega, \bar{\Delta})=P_{\text {in }}^{2}\left|\frac{d R_{\text {out }}}{d u_{m}}\right|^{2} S_{m}(\omega, \bar{\Delta}),
$$

with $d R_{\text {out }} / d u_{m}$ given by Eq. (14). The mechanical PSD is given by the fluctuation dissipation theorem $[1,27]$ :

$$
S_{m}(\omega, \bar{\Delta})=\frac{4 k_{B} T \omega_{m}}{Q_{m}}\left|\chi_{m}^{\mathrm{eff}}(\omega)\right|^{2} .
$$

The full expressions of the optomechanical effects (given in the Appendix) are complex to analyze. Depending on the optical and mechanical characteristics of the system under study, some terms will predominate over others and the full expressions can be simplified. More precisely there are three major properties to consider: (1) the sideband regime defined by the relative difference between the overall optical loss $\bar{\kappa}$ and the mechanical resonance frequency $\omega_{m} ;(2)$ the loss regime defined by the relative difference between the intrinsic and external decay rates, respectively, $\bar{\kappa}_{i}$ and $\bar{\kappa}_{e}$; and (3) the optomechanical coupling configuration defined by the relative difference between the dispersive, the intrinsic, and the external dissipative couplings, respectively, $g_{\mathrm{om}}, \kappa_{\mathrm{om}}^{i}$, and $\kappa_{\mathrm{om}}^{e}$.

All the possible limit cases are represented in Fig. 5. There are 45 limit case situations, characterized by a sideband regime, an optical loss regime, and a coupling configuration, with various impacts on the optical and mechanical dynamics. Each case is accessible experimentally in principle. However, few practical studies have studied the three optomechanical couplings [20-22], and no empirical study on the effects of optical loss and sideband regimes on the optomechanical response has confirmed the possibility of experimentally accessing these regimes. For this reason, and in order to give a physical insight of our model, we choose to analyze one of the practical systems. To this end, we examine several optomechanical configurations in the undercoupled and unresolved sideband regimes and consider the measurements of Tsvirkun et al. on their PhC mechanical resonator suspended over an optical waveguide [22].

The parameters associated with their devices are given in Table II. The mechanical quality factor is chosen in the range $Q_{m} \sim 2000-3000$ (see Tsvirkun et al. [22,31]). The optical decay rates are adjusted according to their measurements. Tsvirkun et al. studied the same mechanical mode (labeled
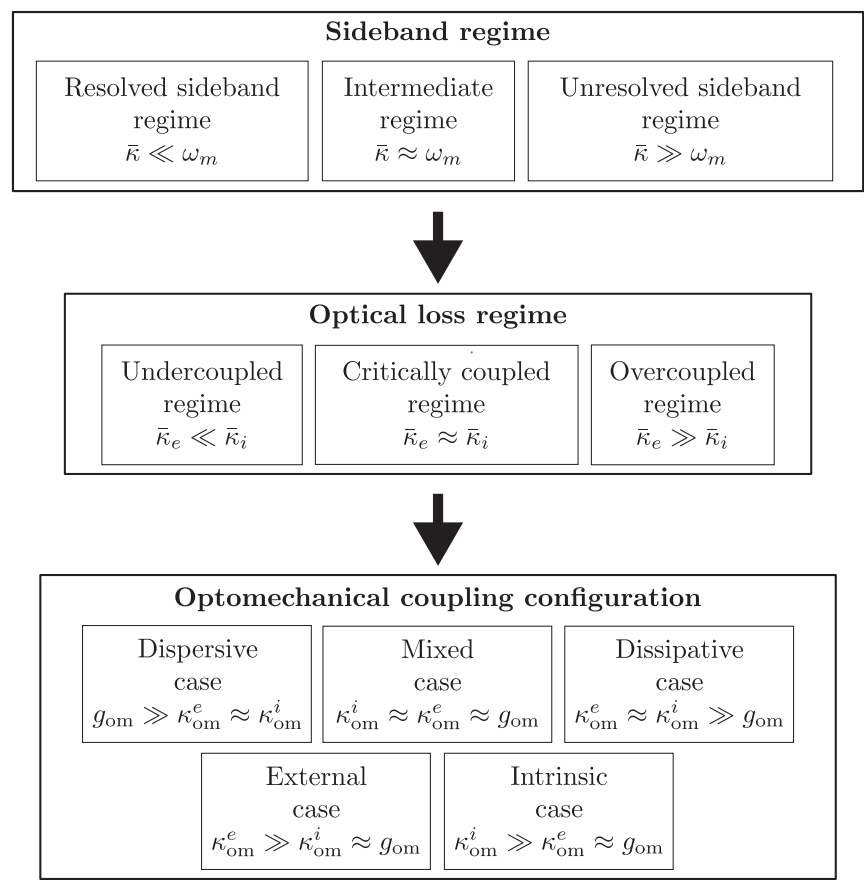

FIG. 5. Schematic representation of limit cases in optomechanical systems with the three types of couplings. There are three properties to consider: the sideband regime, the optical loss regime, and the optomechanical coupling configuration. This leads to 45 limit case situations.

M1) in various configurations depending of the width $w_{\mathrm{wg}}$ of the input waveguide. In order to remain coherent with their measurements, we take into account a proportionality factor in our theoretical optical spectrum and define a corrected optical spectrum $S_{p}$ in $\mathrm{W} / \mathrm{Hz}$ as

$$
S_{p}\left(\omega_{m}, \bar{\Delta}\right)=\frac{\left(\eta \beta^{2} g_{\mathrm{ti}} A\right)^{2}}{R} S_{\mathrm{opt}}\left(\omega_{m}, \bar{\Delta}\right) .
$$

Tsvirkun et al. [22] explain that " $\eta=0.8$ is the coupling efficiency between the laser output and the lens focusing the beam onto the grating coupler, $\beta=0.035$ is the coupling efficiency into (and out of) the access waveguide, $A=25$ is the signal amplification, $g_{\mathrm{ti}}=1400 \mathrm{~V} / \mathrm{W}$ is the transimpedance gain of the photodetector and $R=50 \Omega$ " (see [22] for more details). In the following, $S_{p}$ is considered as the optical PSD for the sake of clarity. We apply our model to these systems and compare it to the measurements in Figs. 6 and 7. The associated dispersive and dissipative optomechanical couplings (from their measurements) are indicated in the figures for each configuration.

The optical spring effect, i.e., the variation of the mechanical resonance frequency with the optical detuning, is compared in Figs. 6(a) (from [22]) and 6(c) (analytical expression). Good agreement is found between our model and the experimental results with a similar detuning dependency and the same order of magnitude of $5 \mathrm{kHz}$ for $\delta \omega_{m}$. This maximum variation of the mechanical resonance frequency occurs close to optical resonance, which is the signature of an important dissipative behavior. 

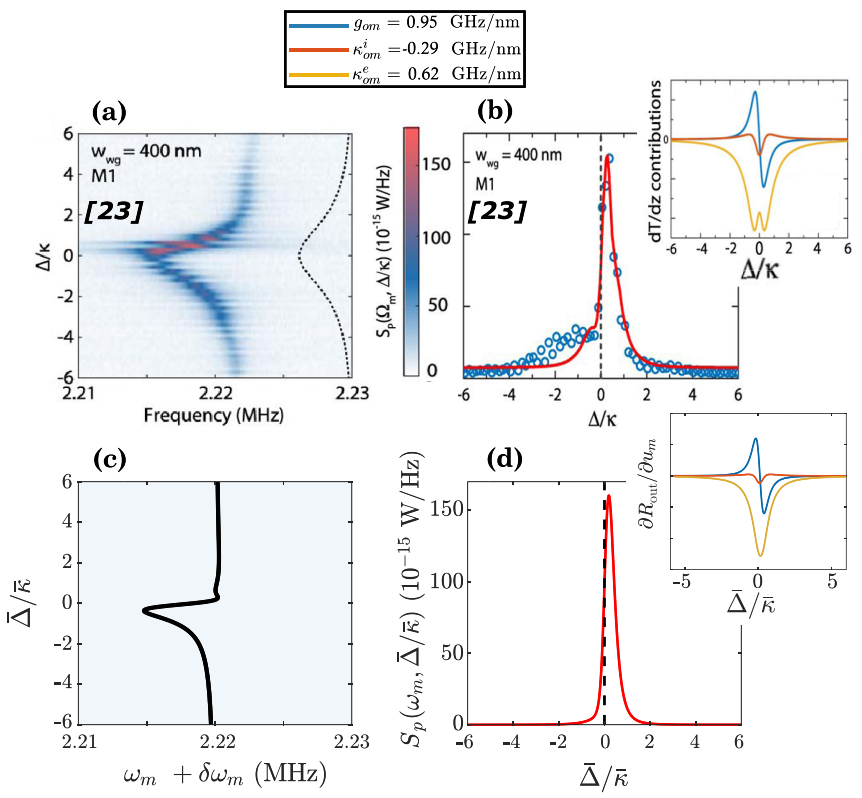

FIG. 6. Optical spring effect [(a) from [22] and (c) from theory] and optical PSD at mechanical resonance frequency $S_{p}\left(\omega_{m}, \bar{\Delta}\right)$ with $\omega_{m}$ depending on $\bar{\Delta}$ [(b) from [22] and (d) from theory] as a function of the normalized optical detuning $\bar{\Delta} / \bar{\kappa}$ for an input waveguide width $w_{\mathrm{wg}}=450 \mathrm{~nm}$. The colorbar of figure (a) is not considered as we are only comparing the variation of the mechanical resonance frequency. The insets show the contribution of each coupling on $d R_{\text {out }} / d u_{m}$ $\left(g_{\text {om }} \partial R_{\text {out }} / \partial \bar{\Delta}\right.$ in blue, $\kappa_{\text {om }}^{i} \partial R_{\text {out }} / \partial \bar{\kappa}_{i}$ in red, and $\kappa_{\text {om }}^{e} \partial R_{\text {out }} / \partial \bar{\kappa}_{e}$ in yellow). Tsvirkun et al. [22] determined it experimentally by fitting the optical spectrum on mechanical resonance with Eq. (14), which allows them to identify the coupling strengths.

The optical PSD at mechanical resonance frequency $S_{p}\left(\omega_{m}, \bar{\Delta}\right.$ ) (with $\omega_{m}$ depending on $\bar{\Delta}$ ) as a function of the normalized optical detuning is compared in Figs. 6(b) (from [22]) and 6(d) (analytical expression). The insets show the contribution of each optomechanical coupling on the mechanically induced optical response oscillations [i.e., the three terms $g_{\text {om }} \partial R_{\text {out }} / \partial \bar{\Delta}, \kappa_{\text {om }}^{i} \partial R_{\text {out }} / \partial \bar{\kappa}_{i}$, and $\kappa_{\text {om }}^{e} \partial R_{\text {out }} / \partial \bar{\kappa}_{e}$, see Eqs. (14)-(16)]. The discrepancy on optical resonance between the insets of measurements and theory for the external dissipative contribution (i.e., $\kappa_{\mathrm{om}}^{e} \partial R_{\mathrm{out}} / \partial \bar{\kappa}_{e}$ ) is due to a Fano modification of the optical response in the experiments. We do not consider this effect as it has no impact on the optomechanical effects [20]. The detuning dependency is governed by the external decay rate regime, i.e., the value of $\bar{\kappa}_{e}$, and the order of magnitude is mostly governed by the decay rates, the input power, and the mechanical properties. As the last two are fixed, the decay rates are adjusted (see Table II). The overall optical loss rate $\bar{\kappa}$ is kept close to $0.1-1 \mathrm{~nm}$ [31]. The external loss rate $\bar{\kappa}_{e}$ is determined by looking at the contribution of each coupling on $d R_{\text {out }} / d u_{m}$ and by comparing it to the measurements of Tsvirkun et al. [22,31] [see insets of Figs. 6(b) and 6(d)]. Good agreement is found in the detuning dependency of the optical PSD on mechanical resonance between measurements and theory, with a single, slightly optically detuned sideband due to the comparable dispersive and external dissipative optomechanical coupling values in the undercoupled regime. The orders of magnitude of the optical PSD are comparable with a maximum close to $150 \mathrm{fW} / \mathrm{Hz}$.

In order to study situations with various optomechanical configurations, we compare multiple optical spectra on mechanical resonance in Fig. 7. Each measurement corresponds to a different input waveguide width. For each situation, we follow the same procedure as before, and find the best $\bar{\kappa}$ and $\overline{\kappa_{e}}$ by comparing the contribution of each coupling on mechanically induced optical response oscillations in theory and in practice. For each optomechanical coupling configuration, the system is in the undercoupled regime, and the corresponding decay rates are given in Table II. Once again, the orders of magnitude of the optical spectra are in good agreement with the measurements, and the detuning dependencies follow the same behaviors, which validates our analytical model.

\section{CONCLUSION}

In this paper, we have extended the theoretical framework used to describe optomechanical systems to the general case of a simultaneously dispersive, external dissipative, and internal dissipative coupling scheme. While the previous theoretical studies did not consider clearly the effect induced by the presence of the three couplings [7,23,24], we have highlighted, by means of a complete description of the mean optical output response, the interest of the three optical loss regimes and the detuning dependency of the mechanically induced optical power oscillations in various optomechanical coupling configurations. The mechanical spectrum and the usual optomechanical effects (optical spring effect and optomechanical damping) have been investigated. In particular, we have revealed the existence of intertwined terms due to "interferences" between the couplings. The optical spectrum has been calculated in a concrete example and comparisons with previous measurements have shown excellent agreement. The study made in this paper can be used in future optomechanical experiments to quantify the three couplings and understand their relative influence on the optical and mechanical responses.

As a conclusion, we want to point out the interest of our model for practical devices. Optomechanical coupling implies a reciprocal dependence of the mechanical response on the optical properties. This effect can be exploited in a sensor, which is even more interesting in the presence of the three couplings. If, for example, a disturbance (a perturbation) of interest induces optical losses, the mechanical properties (effective frequency and effective quality factor) as well as the dissipative coupling values will be affected accordingly. Our model (including the mean optical power oscillations and the description of the mechanical dynamics) can thus allow a better understanding of the mechanisms involved and a proper assessment of the sensitivity of every parameter to such disturbance. We believe all the theoretical tools introduced in this paper will benefit future studies and also serve as modeling tools for designing practical optomechanical devices, such as accelerometers, force sensors, gas spectroscopic and biophotonic sensors, and optical signal processing devices [32]. 

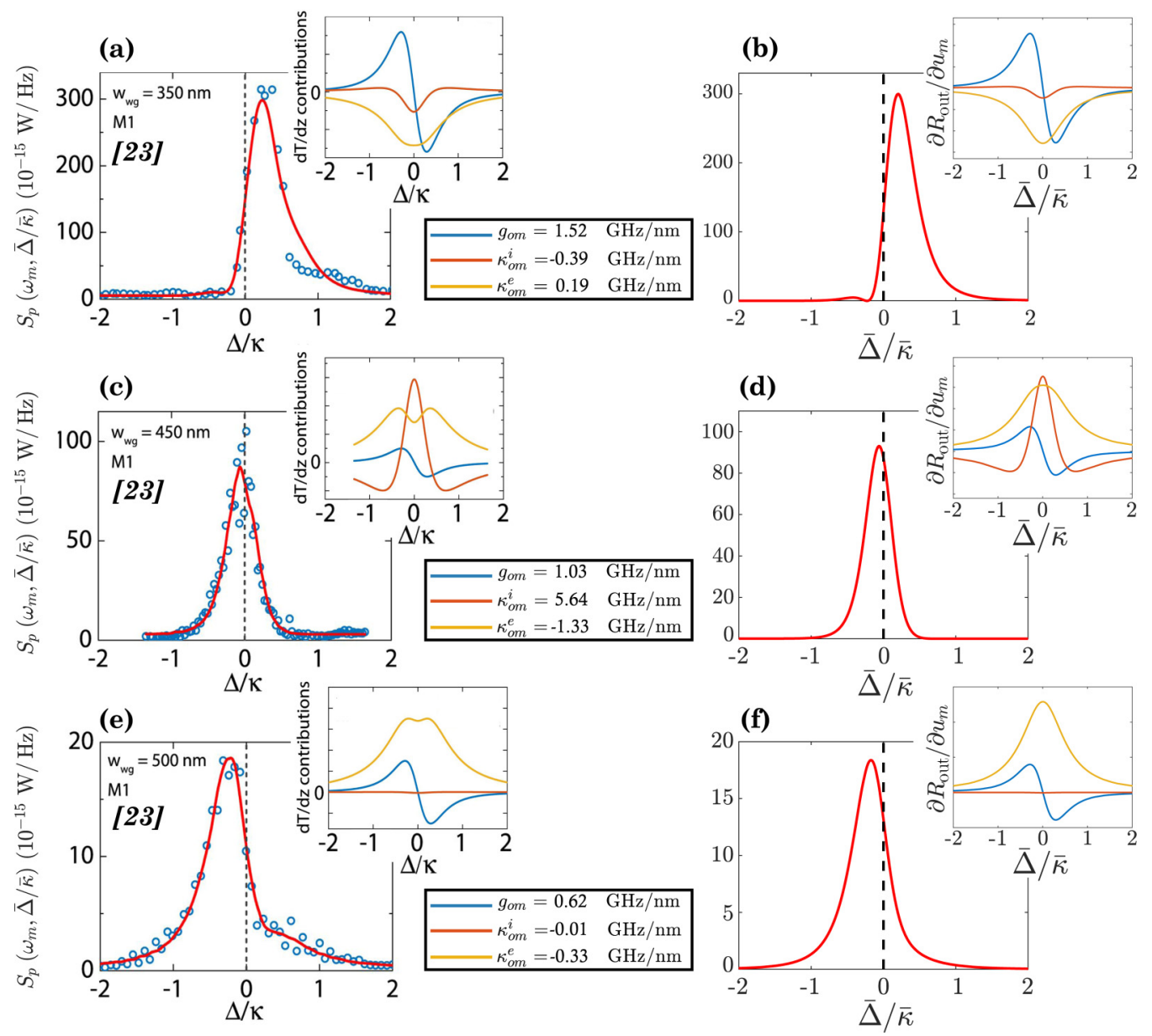

FIG. 7. Optical PSD at mechanical resonance frequency $\left[S_{p}\left(\omega_{m}, \bar{\Delta}\right)\right.$ with $\omega_{m}$ depending on $\left.\bar{\Delta}\right]$ as a function of the normalized optical detuning $\bar{\Delta} / \bar{\kappa}$ for different input waveguide widths: $w_{\mathrm{wg}}=350 \mathrm{~nm}$ [(a) from [22] and (b) from theory], $w_{\mathrm{wg}}=450 \mathrm{~nm}$ [(c) from [22] and (d) from theory], and $w_{\mathrm{wg}}=500 \mathrm{~nm}$ [(e) from [22] and (f) from theory]. The insets show the contribution of each coupling on $d R_{\text {out }} / d u_{m}$ ( $g_{\text {om }} \partial R_{\text {out }} / \partial \bar{\Delta}$ in blue, $\kappa_{\text {om }}^{i} \partial R_{\text {out }} / \partial \bar{\kappa}_{i}$ in red, and $\kappa_{\mathrm{om}}^{e} \partial R_{\text {out }} / \partial \bar{\kappa}_{e}$ in yellow). Note that the discrepancy on optical resonance between the insets of measurements and theory for the external dissipative contribution (i.e., $\kappa_{\mathrm{om}}^{e} \partial R_{\mathrm{out}} / \partial \bar{\kappa}_{e}$ ) is due to a Fano modification of the optical response in practice [20].

\section{APPENDIX: GENERAL EXPRESSIONS OF OPTOMECHANICAL EFFECTS}

The general expressions of each term of the optical spring effect $\delta \omega_{m}$ and optomechanical damping $\gamma_{\text {opt }}$ given, respectively, by Eqs. (30) and (31) are calculated here thanks to the method described in the main paper. The terms due to purely dispersive, external, and intrinsic dissipative situations are given in the following:

$$
\begin{gathered}
\delta \omega_{m}^{\mathrm{disp}}(\omega, \bar{\Delta})=\frac{\hbar g_{\mathrm{om}}^{2}}{2 m_{\mathrm{eff}}} \frac{\bar{n}_{\mathrm{cav}}}{\omega} Q(\omega), \\
\delta \omega_{m}^{\mathrm{diss}, e}(\omega, \bar{\Delta})=\frac{\hbar\left(\kappa_{\mathrm{om}}^{e}\right)^{2}}{16 m_{\mathrm{eff}}} \frac{\bar{n}_{\mathrm{cav}}}{\omega \bar{\kappa}_{e 0}}\left[\frac{\bar{\kappa}\left(\bar{\kappa} / 2-\bar{\kappa}_{e 0}\right)+2 \bar{\Delta}^{2}}{\bar{\kappa}_{e 0}} Q(\omega)-\bar{\kappa} \bar{\Delta} P(\omega)\right], \\
\delta \omega_{m}^{\mathrm{diss}, i}(\omega, \bar{\Delta})=-\frac{\hbar\left(\kappa_{\mathrm{om}}^{i}\right)^{2}}{16 m_{\mathrm{eff}}} \frac{\bar{n}_{\mathrm{cav}} \bar{\kappa}}{\omega \bar{\kappa}_{e 0}}[Q(\omega)+\bar{\Delta} P(\omega)],
\end{gathered}
$$

and

$$
\begin{gathered}
\gamma_{\mathrm{opt}}^{\mathrm{disp}}(\omega, \bar{\Delta})=\frac{\hbar g_{\mathrm{om}}^{2}}{m_{\mathrm{eff}}} \frac{\bar{n}_{\mathrm{cav}}}{\omega} \frac{\bar{\kappa}}{2} S(\omega), \\
\gamma_{\mathrm{opt}}^{\mathrm{diss}, e}(\omega, \bar{\Delta})=\frac{\hbar\left(\kappa_{\mathrm{om}}^{e}\right)^{2}}{4 m_{\mathrm{eff}}} \frac{\bar{n}_{\mathrm{cav}}}{\omega \bar{\kappa}_{e 0}}\left[\frac{\bar{\kappa}^{2}\left(\bar{\kappa} / 2-\bar{\kappa}_{e 0}\right)+2 \bar{\kappa} \bar{\Delta}^{2}}{4 \bar{\kappa}_{e 0}} S(\omega)+\bar{\Delta} R(\omega)\right], \\
\gamma_{\mathrm{opt}}^{\mathrm{diss}, i}(\omega, \bar{\Delta})=\frac{\hbar\left(\kappa_{\mathrm{om}}^{i}\right)^{2}}{4 m_{\mathrm{eff}}} \frac{\bar{n}_{\mathrm{cav}}}{\omega \bar{\kappa}_{e 0}}\left[-\frac{\bar{\kappa}^{2}}{4} S(\omega)+\bar{\Delta} R(\omega)\right]
\end{gathered}
$$


where

$$
\bar{n}_{\mathrm{cav}}=\frac{\bar{\kappa}_{e 0}}{(\bar{\kappa} / 2)^{2}+\bar{\Delta}^{2}} \frac{P_{\mathrm{in}}}{\hbar \omega_{L}}
$$

represents the steady-state intracavity photon number and $P, Q, R$, and $S$ correspond to sums or differences of the Lorentzian shape effective cavity responses $\left|\chi_{\text {cav }}^{\text {eff }}( \pm \omega)\right|^{2}$, with $\chi_{\text {cav }}^{\text {eff }}(\omega)=[\bar{\kappa} / 2-i(\bar{\Delta}+\omega)]^{-1}$, weighted or not with detuning terms $\Delta \pm \omega$ according to

$$
\begin{aligned}
& P(\omega)=\left|\chi_{\mathrm{cav}}^{\mathrm{eff}}(\omega)\right|^{2}+\left|\chi_{\mathrm{cav}}^{\mathrm{eff}}(-\omega)\right|^{2}, \\
& Q(\omega)=(\bar{\Delta}+\omega)\left|\chi_{\mathrm{cav}}^{\mathrm{eff}}(\omega)\right|^{2}+(\bar{\Delta}-\omega)\left|\chi_{\mathrm{cav}}^{\mathrm{eff}}(-\omega)\right|^{2}, \\
& R(\omega)=(\bar{\Delta}+\omega)\left|\chi_{\mathrm{cav}}^{\mathrm{eff}}(\omega)\right|^{2}-(\bar{\Delta}-\omega)\left|\chi_{\mathrm{cav}}^{\mathrm{eff}}(-\omega)\right|^{2}, \\
& S(\omega)=\left|\chi_{\mathrm{cav}}^{\mathrm{eff}}(\omega)\right|^{2}-\left|\chi_{\mathrm{cav}}^{\mathrm{eff}}(-\omega)\right|^{2} .
\end{aligned}
$$

Finally, the intertwined terms linked to "interference" between the couplings are given in the following:

$$
\begin{gathered}
\delta \omega_{m}^{\mathrm{disp}, \mathrm{diss}, e}(\omega, \bar{\Delta})=\frac{\hbar g_{\mathrm{om}} \kappa_{\mathrm{om}}^{e}}{2 m_{\mathrm{eff}}} \frac{\bar{n}_{\mathrm{cav}}}{\omega \bar{\kappa}_{e 0}}\left[-\frac{\bar{\kappa} \bar{\kappa}_{e 0}}{4} P(\omega)+\bar{\Delta} Q(\omega)\right], \\
\delta \omega_{m}^{\mathrm{disp}, \mathrm{diss}, i}(\omega, \bar{\Delta})=\frac{\hbar g_{\mathrm{om}} \kappa_{\mathrm{om}}^{i}}{4 m_{\mathrm{eff}}} \frac{\bar{n}_{\mathrm{cav}}}{\omega \bar{\kappa}_{e 0}}\left[-\frac{\bar{\kappa}\left(\bar{\kappa} / 2+\bar{\kappa}_{e 0}\right)}{2} P(\omega)+\bar{\Delta} Q(\omega)\right], \\
\delta \omega_{m}^{\mathrm{diss}, e, \text { diss }, i}(\omega, \bar{\Delta})=\frac{\hbar \kappa_{\mathrm{om}}^{e} \kappa_{\mathrm{om}}^{i}}{8 m_{\mathrm{eff}}} \frac{\bar{n}_{\mathrm{cav}}}{\omega \bar{\kappa}_{e 0}}\left[\frac{\bar{\kappa}\left(\bar{\kappa} / 2-2 \bar{\kappa}_{e 0}\right)+2 \bar{\Delta}^{2}}{2 \bar{\kappa}_{e 0}} Q(\omega)-\bar{\Delta} \bar{\kappa} P(\omega)\right],
\end{gathered}
$$

and

$$
\begin{gathered}
\gamma_{\mathrm{om}}^{\mathrm{disp}, \mathrm{diss}, e}(\omega, \bar{\Delta})=\frac{\hbar g_{\mathrm{om}} \kappa_{\mathrm{om}}^{e}}{2 m_{\mathrm{eff}}} \frac{\bar{n}_{\mathrm{cav}}}{\omega \bar{\kappa}_{e 0}}\left[\bar{\kappa} \bar{\Delta} S(\omega)+\bar{\kappa}_{e 0} R(\omega)\right], \\
\gamma_{\mathrm{om}}^{\mathrm{disp}, \mathrm{diss}, i}(\omega, \bar{\Delta})=\frac{\hbar g_{\mathrm{om}} \kappa_{\mathrm{om}}^{i}}{4 m_{\mathrm{eff}}} \frac{\bar{n}_{\mathrm{cav}}}{\omega \bar{\kappa}_{e 0}}\left[\bar{\kappa} \bar{\Delta} S(\omega)+\left(2 \bar{\kappa}_{e 0}+\bar{\kappa}\right) R(\omega)\right], \\
\gamma_{\mathrm{om}}^{\mathrm{diss}, e, \mathrm{diss}, i}(\omega, \bar{\Delta})=\frac{\hbar \kappa_{\mathrm{om}}^{e} \kappa_{\mathrm{om}}^{i}}{4 m_{\mathrm{eff}}} \frac{\bar{n}_{\mathrm{cav}}}{\omega \bar{\kappa}_{e 0}}\left[\frac{\left[\bar{\kappa}\left(\bar{\kappa} / 2-2 \bar{\kappa}_{e 0}\right)+2 \bar{\Delta}^{2}\right] \bar{\kappa}}{4 \bar{\kappa}_{e 0}} S(\omega)+2 \bar{\Delta} R(\omega)\right] .
\end{gathered}
$$

[1] M. Aspelmeyer, T. J. Kippenberg, and F. Marquardt, Cavity Optomechanics (Springer-Verlag, Berlin, 2014).

[2] F. Marquardt, J. P. Chen, A. A. Clerk, and S. M. Girvin, Quantum theory of Cavity-Assisted Sideband Cooling of Mechanical Motion, Phys. Rev. Lett. 99, 093902 (2007).

[3] I. Wilson-Rae, N. Nooshi, W. Zwerger, and T. J. Kippenberg, Theory of Ground State Cooling of a Mechanical Oscillator using Dynamical Backaction, Phys. Rev. Lett. 99, 093901 (2007).

[4] A. Jayich, J. Sankey, B. Zwickl, C. Yang, J. Thompson, S. Girvin, A. Clerk, F. Marquardt, and J. Harris, Dispersive optomechanics: A membrane inside a cavity, New J. Phys. 10, 095008 (2008).

[5] L. Ding, C. Baker, P. Senellart, A. Lemaitre, S. Ducci, G. Leo, and I. Favero, High Frequency Gaas Nano-Optomechanical Disk Resonator, Phys. Rev. Lett. 105, 263903 (2010).

[6] J. Chan, A. Safavi-Naeini, J. Hill, S. Meenehan, and O. Painter, Optimized optomechanical crystal cavity with acoustic radiation shield, Appl. Phys. Lett. 101, 081115 (2012).

[7] F. Elste, S. M. Girvin, and A. A. Clerk, Quantum Noise Interference and Backaction Cooling in Cavity Nanomechanics, Phys. Rev. Lett. 102, 207209 (2009).
[8] W. J. Gu, G. X. Li, and Y. P. Yang, Generation of squeezed states in a movable mirror via dissipative optomechanical coupling, Phys. Rev. A 88, 013835 (2013).

[9] A. Kronwald, F. Marquardt, and A. A. Clerk, Arbitrarily large steady-state bosonic squeezing via dissipation, Phys. Rev. A 88, 063833 (2013).

[10] A. K. Tagantsev, I. V. Sokolov, and E. S. Polzik, Dissipative versus dispersive coupling in quantum optomechanics: Squeezing ability and stability, Phys. Rev. A 97, 063820 (2018).

[11] A. Xuereb, R. Schnabel, and K. Hammerer, Dissipative Optomechanics in a Michelson-Sagnac Interferometer, Phys. Rev. Lett. 107, 213604 (2011).

[12] S. P. Tarabrin, H. Kaufer, F. Y. Khalili, R. Schnabel, and K. Hammerer, Anomalous dynamic backaction in interferometers, Phys. Rev. A 88, 023809 (2013).

[13] A. Sawadsky, H. Kaufer, R. M. Nia, S. P. Tarabrin, F. Y. Khalili, K. Hammerer, and R. Schnabel, Observation of Generalized Optomechanical Coupling and Cooling on Cavity Resonance, Phys. Rev. Lett. 114, 043601 (2015).

[14] A. Nazmiev and S. P. Vyatchanin, Stable optical rigidity based on dissipative coupling, J. Phys. B 52, 155401 (2019). 
[15] M. Li, W. H. P. Pernice, and H. X. Tang, Reactive Cavity Optical Force on Microdisk-Coupled Nanomechanical Beam Waveguides, Phys. Rev. Lett. 103, 223901 (2009).

[16] S. Huang and G. S. Agarwal, Reactive-coupling-induced normal mode splittings in microdisk resonators coupled to waveguides, Phys. Rev. A 81, 053810 (2010).

[17] C. Fu, K. Gu, X. Yan, X. Yang, C. Cui, and J. Wu, Normal mode splitting due to quadratic reactive coupling in a microdiskwaveguide optomechanical system, Phys. Lett. A 377, 133 (2012).

[18] R. Madugani, Y. Yang, J. Ward, V. Le, and S. N. Chormaic, Optomechanical transduction and characterization of a silica microsphere pendulum via evanescent light, Appl. Phys. Lett. 106, 241101 (2015).

[19] J. G. Huang, Y. Li, L. K. Chin, H. Cai, Y. D. Gu, M. F. Karim, J. H. Wu, T. N. Chen, Z. C. Yang, Y. L. Hao, C. W. Qiu, and A. Q. Liu, A dissipative self-sustained optomechanical resonator on a silicon chip, Appl. Phys. Lett. 112, 051104 (2018).

[20] M. Wu, A. C. Hryciw, C. Healey, D. P. Lake, H. Jayakumar, M. R. Freeman, J. P. Davis, and P. E. Barclay, Dissipative and Dispersive Optomechanics in a Nanocavity Torque Sensor, Phys. Rev. X 4, 021052 (2014).

[21] A. C. Hryciw, M. Wu, B. Khanaliloo, and P. E. Barclay, Tuning of nanocavity optomechanical coupling using a near-field fiber probe, Optica 2, 491 (2015).

[22] V. Tsvirkun, A. Surrente, F. Raineri, G. Beaudoin, R. Raj, I. Sagnes, I. Robert-Philip, and R. Braive, Integrated III-V photonic crystal: $\mathrm{Si}$ waveguide platform with tailored optomechanical coupling, Sci. Rep. 5, 1 (2015).
[23] T. Weiss, C. Bruder, and A. Nunnenkamp, Strong-coupling effects in dissipatively coupled optomechanical systems, New J. Phys. 15, 045017 (2013).

[24] T. Weiss and A. Nunnenkamp, Quantum limit of laser cooling in dispersively and dissipatively coupled optomechanical systems, Phys. Rev. A 88, 023850 (2013).

[25] O. Arcizet, P.-F. Cohadon, T. Briant, M. Pinard, and A. Heidmann, Radiation-pressure cooling and optomechanical instability of a micromirror, Nature (London) 444, 71 (2006).

[26] C. W. Gardiner and P. Zoller, Quantum Noise (Springer-Verlag, Berlin, 2004).

[27] B. Hauer, C. Doolin, K. Beach, and J. Davis, A general procedure for thermomechanical calibration of nano/micro-mechanical resonators, Ann. Phys. (NY) 339, 181 (2013).

[28] R. Ghobadi, A. R. Bahrampour, and C. Simon, Quantum optomechanics in the bistable regime, Phys. Rev. A 84, 033846 (2011).

[29] E. A. Sete and H. Eleuch, Controllable nonlinear effects in an optomechanical resonator containing a quantum well, Phys. Rev. A 85, 043824 (2012).

[30] O. Kyriienko, T. C. H. Liew, and I. A. Shelykh, Optomechanics with Cavity Polaritons: Dissipative Coupling and Unconventional Bistability, Phys. Rev. Lett. 112, 076402 (2014).

[31] V. Tsvirkun, Optomechanics in hybrid fully-integrated twodimensional photonic crystal resonators, Ph.D. thesis, ParisSaclay University, 2015.

[32] M. Metcalfe, Applications of cavity optomechanics, Appl. Phys. Rev. 1, 031105 (2014). 